\title{
EXISTENCE AND UNIQUENESS FOR THE LINEAR KOITER MODEL FOR SHELLS WITH LITTLE REGULARITY
}

\author{
BY
}

ADEL BLOUZA AND HERVÉ LE DRET

Laboratoire d'Analyse Numérique, Université Pierre et Marie Curie, 75252 Paris Cedex 05, France

\begin{abstract}
We give a simple proof of existence and uniqueness of the solution of the Koiter model for linearly elastic thin shells whose midsurfaces can have charts with discontinuous second derivatives. The proof is based on new expressions for the linearized strain and change of curvature tensors. It also makes use of a new version of the rigid displacement lemma under hypotheses of regularity for the displacement and the midsurface of the shell that are weaker than those required by earlier proofs.

Résumé. On donne une démonstration simple de l'existence et l'unicité de la solution du modèle de Koiter pour des coques minces linéairement élastiques dont les surfaces moyennes peuvent avoir des dérivées secondes discontinues. La démonstration est fondée sur de nouvelles expressions des tenseurs linéarisés de déformation et de changement de courbure. Elle utilise également une version nouvelle du lemme du mouvement rigide pour une coque, sous des hypothèses de régularité du déplacement et de la surface moyenne plus faibles que celles des démonstrations antérieures.
\end{abstract}

1. Introduction. There exist at least two different families of linear models for thin elastic shells: the one of Reissner, which relies on the theory of Cosserat surfaces, cf. Cosserat and Cosserat [10], and the Kirchhoff-Love type theories. The second approach is based on the celebrated Kirchhoff-Love assumptions, which state that the normals to the reference midsurfaces are deformed into normals to the deformed midsurface and that the distance between a point and the midsurface remains constant throughout the deformation of the shell.

Taking into account these assumptions, Koiter [17] proposed a two-dimensional mathematical model for linearly elastic thin shells where the unknown is the displacement field of the points of the shell midsurface. An approximation for the displacement field across the thickness of the shell may be derived from this displacement via the Kirchhoff-Love assumptions. We refer to Bernadou [1] for a recent overview of linear shell theory.

Received December 10, 1996.

1991 Mathematics Subject Classification. Primary 73K15.

This work is part of the HCM program "Shells: Mathematical Modeling and Analysis, Scientific Computing" of the Commission of the European Communities (contract ERBCHRXCT940536). 
An existence and uniqueness theorem for Koiter's model was established for the first time by Bernadou and Ciarlet [2] by means of a particularly technical proof relying on results of Rougée [19]. Applying a lemma on distributions in $H^{-1}$ whose gradient is also in $H^{-1}$ of J.-L. Lions, Ciarlet and Miara [9] were able to give a simpler existence and uniqueness proof.

The purpose of this work is to provide an even simpler proof of existence and uniqueness for Koiter's model. Moreover, our result is valid for shells whose midsurface can have charts with discontinuous second derivatives. We thus improve-and significantly simplify - the earlier proofs of Bernadou and Ciarlet [2], Ciarlet and Miara [9], and Bernadou, Ciarlet and Miara [3], which all assumed midsurfaces of class at least $C^{3}$.

The article is as follows. We start in Sec. 2 with a brief review of some notions of differential geometry that will be used thereafter. We then introduce in Sec. 3 expressions for the linearized strain and change of curvature tensors of a displacement of the midsurface. To the best of our knowledge, these expressions are new or at least previously unnoticed in this context, especially as concerns the change of curvature tensor. The crucial point for our purposes here is that they do not involve any derivatives of the second fundamental form of the midsurface. Such derivatives thus do not actually enter in the change of curvature tensor, contrarily to what was thought previously. It is this observation that allows us to weaken the customary regularity requirements for the midsurface. Indeed, the new expressions are valid for midsurfaces of class $W^{2, \infty}$ and are defined in general as distributions. In order to obtain these new expressions, we do not decompose the displacement on the contravariant basis as is usually done. Instead, we simply consider displacements as $\mathbb{R}^{3}$-valued functions, which is indeed a more intrinsic approach. A further consequence of this approach is that our expressions for the strain and change of curvature tensors are considerably simpler than the classical ones.

In Sec. 4, we use these new expressions to prove the rigid displacement lemma for a shell, viz. Lemma 5, under hypotheses of regularity for the displacement and midsurface that are significantly weaker than those required by earlier proofs. More precisely, the midsurface is only of class $W^{2, \infty}$ and the displacement of class $H^{1}$. The proof is based on the existence of the infinitesimal rotation vector $\psi$; see Vekua [21] for the classical approach, which follows from elementary arguments of vector analysis in $\mathbb{R}^{3}$ recast in a distributional framework.

In Sec. 5, we establish the ellipticity of the bilinear form associated with the Koiter model over an appropriate Hilbert space in the case of simple support on the boundary. The proof uses the standard contradiction argument, based on the one hand on the twodimensional Korn inequality and on the other hand on Rellich's lemma. The existence and uniqueness result then follows from the Lax-Milgram lemma. Again, this is made possible by our new expressions for the linearized strain and change of curvature tensors.

We show in Sec. 6 that our method also works for the case of clamping on one part of the boundary and applied forces and moments on the remaining part. The general philosophy at work here is the same as before. We rewrite the clamping condition in a more intrinsic way than the classical one by considering the displacements as $\mathbb{R}^{3}$-valued functions and not as triples of covariant components. It turns out that the resulting expressions make sense in our functional framework. We proceed in a similar fashion 
for the infinitesimal rotation vector, which is used to write down the loading terms corresponding to moments applied on the boundary. The resulting expressions are once again simpler and more natural than the classical ones, and they make sense in our functional framework. Let us note that it is especially interesting insofar as it allows quite common situations, such as a $C^{1}$-shell made of a plane part and a smooth cylindrical part, which are excluded by the usual hypothesis that the midsurface be of class $C^{3}$.

Finally, Sec. 7 is devoted to a comparison with existing results in the literature, a discussion of numerical issues and a few examples and applications.

Let us sum up by emphasizing again that the main novelty of this article, in addition to a significant simplification of the proofs, is that it allows shells whose midsurfaces may have curvature discontinuities. Note that Destuynder and Salaün [13], [14] obtained a mixed formulation of the Koiter model that is also valid for a $W^{2, \infty}$ shell in that thirdorder derivatives of the chart do not appear in the final model. However, the existence of third-order derivatives seems to be required to derive their formulation and existence and uniqueness are obtained as a consequence of Bernadou and Ciarlet's result; see the discussion at the end of this article. The idea of foregoing covariant components was also used by Le Tallec and coworkers, see e.g. [18], in the linear and nonlinear cases. Part of the results of the present article were announced in Blouza and Le Dret [5], [6].

2. Geometry of the shell midsurface. In the sequel, Greek indices and exponents always belong to the set $\{1,2\}$, while Latin indices and exponents belong to the set $\{1,2,3\}$. We use the Einstein summation convention, unless otherwise specified.

Let $\left(e_{1}, e_{2}, e_{3}\right)$ be the orthonormal canonical basis of the Euclidean space $\mathbb{R}^{3}$. We denote by $u \cdot v$ the inner product of $\mathbb{R}^{3},|u|=\sqrt{u \cdot u}$ the associated Euclidean norm, and $u \wedge v$ the vector product of $u$ and $v$.

Let $\omega$ denote a Lipschitz domain of $\mathbb{R}^{2}$. We consider a shell of midsurface $S=\varphi(\bar{\omega})$, where $\varphi \in W^{2, \infty}\left(\omega ; \mathbb{R}^{3}\right)$ is an injective mapping such that the two vectors

$$
a_{\alpha}(x)=\partial_{\alpha} \varphi(x)
$$

are linearly independent at each point $x \in \bar{\omega}$. We let

$$
a_{3}(x)=\frac{a_{1}(x) \wedge a_{2}(x)}{\left|a_{1}(x) \wedge a_{2}(x)\right|}
$$

be the unit normal vector on the midsurface at point $\varphi(x)$. The vectors $a_{i}(x)$ define the covariant basis at point $\varphi(x)$. The regularity of the midsurface chart and the hypothesis of linear independence on $\bar{\omega}$ imply that the vectors $a_{i}$ belong to $W^{1, \infty}\left(\omega ; \mathbb{R}^{3}\right)$. The contravariant basis $a^{i}(x)$ is defined by the relations

$$
a^{i}(x) \cdot a_{j}(x)=\delta_{j}^{i}
$$

where $\delta_{j}^{i}$ is the Kronecker symbol. In particular, $a^{3}(x)=a_{3}(x)$. As before, $a^{i} \in$ $W^{1, \infty}\left(\omega ; \mathbb{R}^{3}\right)$. We let $a(x)=\left|a_{1}(x) \wedge a_{2}(x)\right|^{2}$, so that $\sqrt{a}$ is the area element of the midsurface in the chart $\varphi$. 
The first and second fundamental forms of the surface are given in covariant components by

$$
a_{\alpha \beta}=a_{\alpha} \cdot a_{\beta} \quad \text { and } \quad b_{\alpha \beta}=a_{3} \cdot \partial_{\beta} a_{\alpha}=-a_{\alpha} \cdot \partial_{\beta} a_{3} .
$$

Since $W^{1, \infty}\left(\omega ; \mathbb{R}^{3}\right)$ is a Banach algebra, it follows that $a_{\alpha \beta} \in W^{1, \infty}(\omega)$ and $b_{\alpha \beta} \in L^{\infty}(\omega)$. We further introduce the contravariant components of the first fundamental form

$$
a^{\alpha \beta}=a^{\alpha} \cdot a^{\beta}
$$

and the mixed components of the second fundamental form

$$
b_{\alpha}^{\beta}=a^{\beta \rho} b_{\rho \alpha} .
$$

Again, $a^{\alpha \beta} \in W^{1, \infty}(\omega)$ and $b_{\alpha}^{\beta} \in L^{\infty}(\omega)$. Finally, the Christoffel symbols of the midsurface are given by

$$
\Gamma_{\alpha \beta}^{\rho}=\Gamma_{\beta \alpha}^{\rho}=a^{\rho} \cdot \partial_{\beta} a_{\alpha}
$$

and we have $\Gamma_{\alpha \beta}^{\rho} \in L^{\infty}(\omega)$.

3. The linearized strain and change of curvature tensors. In this section, we define the linearized strain and change of curvature tensors of a shell displacement in a functional framework that is weaker than the usual one.

We begin by recalling the classical definitions. Assume thus that $\varphi \in C^{3}\left(\bar{\omega} ; \mathbb{R}^{3}\right)$. Let $u$ be a displacement of the midsurface, i.e., a regular mapping from $\bar{\omega}$ into $\mathbb{R}^{3}$ given in covariant components by $u(x)=u_{i}(x) a^{i}(x)$ where $u_{i}=u \cdot a_{i}$. In the classical approach, the displacement is identified with the triple $\left(u_{i}\right), i=1,2,3$, of its covariant components. The covariant derivatives of the tangential components of $u$ are defined as

$$
u_{\alpha \mid \beta}=\partial_{\beta} u_{\alpha}-\Gamma_{\alpha \beta}^{\rho} u_{\rho}
$$

its linearized strain tensor is given by $\gamma(u)=\gamma_{\alpha \beta}(u) a^{\alpha} \otimes a^{\beta}$ with

$$
\gamma_{\alpha \beta}(u)=\frac{1}{2}\left(u_{\alpha \mid \beta}+u_{\beta \mid \alpha}\right)-b_{\alpha \beta} u_{3}
$$

and its linearized change of curvature tensor by $\Upsilon(u)=\Upsilon_{\alpha \beta}(u) a^{\alpha} \otimes a^{\beta}$ with

$$
\Upsilon_{\alpha \beta}(u)=u_{3 \mid \alpha \beta}-b_{\alpha \rho} b_{\beta}^{\rho} u_{3}+b_{\beta}^{\rho} u_{\rho \mid \alpha}+b_{\alpha}^{\rho} u_{\rho \mid \beta}+b_{\beta \mid \alpha}^{\rho} u_{\rho},
$$

where $u_{3 \mid \alpha \beta}=\partial_{\alpha \beta} u_{3}-\Gamma_{\alpha \beta}^{\rho} \partial_{\rho} u_{3}$ and $b_{\beta \mid \alpha}^{\rho}=\partial_{\alpha} b_{\beta}^{\rho}+\Gamma_{\alpha \sigma}^{\rho} b_{\beta}^{\sigma}-\Gamma_{\beta \alpha}^{\sigma} b_{\sigma}^{\rho}$. It is this last definition that restricts the regularity of the chart in the classical approach. Indeed, in Bernadou and Ciarlet [2] and all subsequent works, the tangential components of the displacement $u_{\rho}$ belong to $H^{1}(\omega)$. For the term $\partial_{\alpha} b_{\beta}^{\rho} u_{\rho}$ to make sense, the above-mentioned authors are led to assume that $\varphi$ is of class $C^{3}$, an assumption that can be slightly relaxed but not essentially so.

We now change points of view and instead of identifying the displacement $u$ with its covariant components, we consider it as a mapping from $\omega$ into $\mathbb{R}^{3}$. Note that this point of view is not entirely intrinsic, since we are still viewing the displacement through the chart $\varphi$ and not as an object defined on the surface itself. See Destuynder [11], Valid [20], for an intrinsic formulation on the surface. In our approach, the partial derivatives $\partial_{\alpha} u$ and $\partial_{\alpha \beta} u$ are again mappings from $\omega$ into $\mathbb{R}^{3}$.

We begin with a density lemma. 
Lemma 1. Let $\varphi \in W^{2, \infty}\left(\omega ; \mathbb{R}^{3}\right)$ be such that $\left|a_{1} \wedge a_{2}\right|>0$ on $\bar{\omega}$. Then there exists a sequence $\varphi^{n} \in C^{3}\left(\bar{\omega} ; \mathbb{R}^{3}\right)$ such that $\left|a_{1}^{n} \wedge a_{2}^{n}\right|>0$ on $\bar{\omega}, \varphi^{n} \rightarrow \varphi$ strongly in $W^{2, p}\left(\omega ; \mathbb{R}^{3}\right)$ for all $1 \leq p<+\infty$ and $\varphi^{n}-\varphi$ weakly-* in $W^{2, \infty}\left(\omega ; \mathbb{R}^{3}\right)$.

Proof. Since $\omega$ is a Lipschitz domain of $\mathbb{R}^{2}$, we may extend $\varphi$ to a function of $W^{2, \infty}\left(\mathbb{R}^{2}\right.$; $\left.\mathbb{R}^{3}\right)$, still denoted $\varphi$. Consider now a sequence of mollifiers $\rho^{n} \in C^{\infty}\left(\mathbb{R}^{2}\right)$ and let $\varphi^{n}=$ $\rho^{n} * \varphi \in C^{\infty}\left(\mathbb{R}^{2} ; \mathbb{R}^{3}\right)$. It is well known that the restriction of $\varphi^{n}$ to $\bar{\omega}$ converges strongly in $C^{1}\left(\bar{\omega} ; \mathbb{R}^{3}\right)$ to $\varphi$ as $n \rightarrow+\infty$, since $W^{2, \infty}\left(\mathbb{R}^{2} ; \mathbb{R}^{3}\right) \hookrightarrow C^{1}\left(\mathbb{R}^{2} ; \mathbb{R}^{3}\right)$. Consequently, for $n$ large enough, $\left|a_{1}^{n} \wedge a_{2}^{n}\right|>0$ on $\bar{\omega}$. The convergence in $W^{2, p}\left(\omega ; \mathbb{R}^{3}\right), 1 \leq p \leq+\infty$, is classical.

LEMmA 2. If $u \in H^{1}\left(\omega ; \mathbb{R}^{3}\right)$ and $\varphi \in W^{2, \infty}\left(\omega ; \mathbb{R}^{3}\right)$, then the expressions

$$
\gamma_{\alpha \beta}^{\text {new }}(u)=\frac{1}{2}\left(\partial_{\alpha} u \cdot a_{\beta}+\partial_{\beta} u \cdot a_{\alpha}\right)
$$

define functions of $L^{2}(\omega)$ that coincide with the covariant components of the strain tensor when $u$ and $\varphi$ belong to $C^{3}\left(\bar{\omega} ; \mathbb{R}^{3}\right)$. The expressions

$$
\Upsilon_{\alpha \beta}^{\text {new }}(u)=\left(\partial_{\alpha \beta} u-\Gamma_{\alpha \beta}^{\rho} \partial_{\rho} u\right) \cdot a_{3}
$$

define distributions of $H^{-1}(\omega)$ that coincide with the covariant components of the change of curvature tensor when $u$ and $\varphi$ belong to $C^{3}\left(\bar{\omega} ; \mathbb{R}^{3}\right)$. Moreover, if $u^{n}$ and $\varphi^{n}$ belong to $C^{3}\left(\bar{\omega} ; \mathbb{R}^{3}\right)$ and are such that $u^{n} \rightarrow u$ strongly in $H^{1}\left(\omega ; \mathbb{R}^{3}\right)$ and $\varphi^{n} \rightarrow \varphi$ strongly in $W^{2, p}\left(\omega ; \mathbb{R}^{3}\right)$ and $\varphi^{n} \rightarrow \varphi$ weakly-* in $W^{2, \infty}\left(\omega ; \mathbb{R}^{3}\right)$, then $\gamma_{\alpha \beta}\left(u^{n}\right) \rightarrow \gamma_{\alpha \beta}^{\text {new }}(u)$ strongly in $L^{2}(\omega)$ and $\Upsilon_{\alpha \beta}\left(u^{n}\right) \rightarrow \Upsilon_{\alpha \beta}^{\text {new }}(u)$ strongly in $H^{-1}(\omega)$

REMARKS. Lemma 2 gives two expressions for the linearized strain and change of curvature tensors that are simpler and more intrinsic than the classical expressions (9) and (10). Note in particular that the derivatives of the second fundamental form are absent from the definition of the change of curvature tensor. In view of Lemma 1, expressions (11) and (12) thus provide natural extensions for the strain and change of curvature tensors to our less smooth situation. We will thus remove the "new" exponent from the notation after the following proof. Note also that the strain and change of curvature tensors depend on the sequence of charts, which is not apparent in the notation.

Proof. Let us be given $u$ and $\varphi$, two elements of $C^{3}\left(\bar{\omega} ; \mathbb{R}^{3}\right)$ such that $\left|a_{1} \wedge a_{2}\right|>0$ on $\bar{\omega}$. Consider the one-parameter family of deformed surfaces $\{(\varphi+\eta u)(\omega), \eta \in \mathbb{R}\}$ for $\eta$ small enough. To obtain the linearized strain and change of curvature tensors of the displacement $u$ with respect to the surface $\varphi(\omega)$, we differentiate the covariant components of the first and second fundamental forms of this family with respect to $\eta$ at $\eta=0$.

i) Let $a_{\alpha}(\eta)=a_{\alpha}+\eta \partial_{\alpha} u$ and $a_{\alpha \beta}(\eta)=a_{\alpha}(\eta) \cdot a_{\beta}(\eta)$. The covariant components of the strain tensor are equal to one half of the covariant components of the first variation of the metric tensor and are thus given by

$$
\gamma_{\alpha \beta}(u)=\frac{1}{2} \frac{d a_{\alpha \beta}}{d \eta}(0) .
$$

It is obvious that

$$
\frac{d a_{\alpha \beta}}{d \eta}(0)=\frac{d a_{\alpha}}{d \eta}(0) \cdot a_{\beta}(0)+a_{\alpha}(0) \cdot \frac{d a_{\beta}}{d \eta}(0)=\partial_{\alpha} u \cdot a_{\beta}+a_{\alpha} \cdot \partial_{\beta} u
$$


hence formula (11). It is easily checked that formula (11) coincides with the classical definition (9) for $u$ and $\varphi$ smooth.

Let us now assume that $u$ and $\varphi$ satisfy the hypotheses of Lemma 2, i.e., $u \in H^{1}\left(\omega ; \mathbb{R}^{3}\right)$ and $\varphi \in W^{2, \infty}\left(\omega ; \mathbb{R}^{3}\right)$. It follows then that $\partial_{\alpha} u \in L^{2}\left(\omega ; \mathbb{R}^{3}\right)$ and $a_{\beta} \in L^{\infty}\left(\omega ; \mathbb{R}^{3}\right)$. Consequently, $\partial_{\alpha} u \cdot a_{\beta} \in L^{2}(\omega)$, which shows that $\gamma_{\alpha \beta}^{\text {new }}(u) \in L^{2}(\omega)$. Finally, it is clear that if $u^{n} \rightarrow u$ and $\varphi^{n} \rightarrow \varphi$ respectively in $H^{1}\left(\omega ; \mathbb{R}^{3}\right)$ and $W^{2, p}\left(\omega ; \mathbb{R}^{3}\right)$ for some $p>2$, then $\gamma_{\alpha \beta}^{\text {new }}\left(u^{n}\right) \rightarrow \gamma_{\alpha \beta}^{\text {new }}(u)$ in $L^{2}(\omega)$ strong. This holds true in particular when $u^{n}$ and $\varphi^{n}$ are regular.

ii) We now turn to the derivation of the change of curvature tensor. Let $A(\eta)=$ $a_{1}(\eta) \wedge a_{2}(\eta)$ so that $a_{3}(\eta)=A(\eta) /|A(\eta)|$ is the deformed normal vector (this is for $\eta$ small enough). The second fundamental form of the deformed surface is $b_{\alpha \beta}(\eta)=$ $\partial_{\beta} a_{\alpha}(\eta) \cdot a_{3}(\eta)$. The covariant components of the change of curvature tensor are the first variation of the covariant components of the second fundamental form and are thus given by

$$
\Upsilon_{\alpha \beta}(u)=\frac{d b_{\alpha \beta}}{d \eta}(0)
$$

Let us compute this derivative. It follows from Leibniz' rule that

$$
\frac{d b_{\alpha \beta}}{d \eta}(0)=\partial_{\alpha \beta} u \cdot a_{3}+\partial_{\beta} a_{\alpha} \cdot \frac{d a_{3}}{d \eta}(0) .
$$

To compute the derivative of the normal vector, we first remark that $d A / d \eta(0)=a_{1} \wedge$ $\partial_{2} u+\partial_{1} u \wedge a_{2}$ and $d|A| / d \eta(0)=(d A / d \eta(0)) \cdot a_{3}$. Therefore,

$$
\frac{d a_{3}}{d \eta}(0)=\frac{1}{|A(0)|}\left(\frac{d A}{d \eta}(0)-\frac{d|A|}{d \eta}(0) a_{3}\right)=\frac{1}{|A(0)|}\left(\frac{d A}{d \eta}(0) \cdot a_{\rho}\right) a^{\rho} .
$$

Note now that $d A / d \eta(0) \cdot a_{\rho}=-A(0) \cdot \partial_{\rho} u$. Consequently,

$$
\frac{d a_{3}}{d \eta}(0)=-\left(\frac{A(0)}{|A(0)|} \cdot \partial_{\rho} u\right) a^{\rho}=-\left(a_{3} \cdot \partial_{\rho} u\right) a^{\rho} .
$$

Replacing this expression into (16), we obtain

$$
\frac{d b_{\alpha \beta}}{d \eta}(0)=\partial_{\alpha \beta} u \cdot a_{3}-\left(a^{\rho} \cdot \partial_{\beta} a_{\alpha}\right)\left(\partial_{\rho} u \cdot a_{3}\right) .
$$

In view of the definition of the Christoffel symbols (7), Eq. (19) becomes

$$
\frac{\partial b_{\alpha \beta}}{\partial \eta}(0)=\left(\partial_{\alpha \beta} u-\Gamma_{\alpha \beta}^{\rho} \partial_{\rho} u\right) \cdot a_{3} .
$$

The above expression for the change of curvature tensor is valid for smooth $u$ and $\varphi$, and it can be checked by a straightforward albeit lengthy computation that it coincides in this case with the classical definition (10).

Let now $u$ and $\varphi$ satisfy the hypotheses of Lemma 2. We remark that expression (12) defines an element of $H^{-1}(\omega)$. Indeed, if $u \in H^{1}\left(\omega ; \mathbb{R}^{3}\right)$ and $\varphi \in W^{2 . \infty}\left(\omega ; \mathbb{R}^{3}\right)$, $\Gamma_{\alpha \beta}^{\rho} \partial_{\rho} u \cdot a_{3}$ belongs trivially to $L^{2}(\omega)$ on the one hand, and on the other hand, $\partial_{\alpha \beta} u \cdot a_{3}$ is a distribution of $H^{-1}(\omega)$ defined by

$$
\forall \theta \in H_{0}^{1}(\omega), \quad\left\langle\partial_{\alpha \beta} u \cdot a_{3}, \theta\right\rangle=\left\langle\partial_{\alpha \beta} u, \theta a_{3}\right\rangle=-\int_{\omega} \partial_{\alpha} u \cdot \partial_{\beta}\left(\theta a_{3}\right) d x .
$$


In effect, since $a_{3} \in W^{1, \infty}\left(\omega ; \mathbb{R}^{3}\right)$, for all $\theta \in H_{0}^{1}(\omega), \theta a_{3}$ belongs to $H_{0}^{1}\left(\omega ; \mathbb{R}^{3}\right)$ and the second duality pairing is well defined as the rightmost integral.

Finally, let $u^{n} \rightarrow u$ in $H^{1}\left(\omega ; \mathbb{R}^{3}\right), \varphi^{n} \rightarrow \varphi$ in $W^{2, p}\left(\omega ; \mathbb{R}^{3}\right)$ for all $p<+\infty$ and $\varphi^{n} \rightarrow \varphi$ weakly-* in $W^{2, \infty}\left(\omega ; \mathbb{R}^{3}\right)$. Then, $\left(a^{\rho}\right)^{n} \rightarrow a^{\rho}$ in $C^{0}\left(\bar{\omega} ; \mathbb{R}^{3}\right), \partial_{\beta} a_{\alpha}^{n} \rightarrow \partial_{\beta} a_{\alpha}$ in $L^{p}\left(\omega ; \mathbb{R}^{3}\right)$, and $\partial_{\beta} a_{\alpha}^{n} \rightarrow \partial_{\beta} a_{\alpha}$ weakly-* in $L^{\infty}\left(\omega ; \mathbb{R}^{3}\right)$. Therefore, $\left(\Gamma_{\alpha \beta}^{\rho}\right)^{n} \rightarrow \Gamma_{\alpha \beta}^{\rho}$ in $L^{p}(\omega)$ and $\left(\Gamma_{\alpha \beta}^{\rho}\right)^{n} \rightarrow \Gamma_{\alpha \beta}^{\rho}$ weakly-* in $L^{\infty}\left(\omega ; \mathbb{R}^{3}\right)$. Since $\partial_{\rho} u^{n} \rightarrow \partial_{\rho} u$ strongly in $L^{2}\left(\omega ; \mathbb{R}^{3}\right)$ and $a_{3}^{n} \rightarrow a_{3}$ strongly in $C^{0}\left(\bar{\omega} ; \mathbb{R}^{3}\right)$, we see that $\left(\Gamma_{\alpha \beta}^{\rho}\right)^{n} \partial_{\rho} u^{n} \cdot a_{3}^{n} \rightarrow \Gamma_{\alpha \beta}^{\rho} \partial_{\rho} u \cdot a_{3}$ in $L^{q}(\omega)$ for $q=2 p /(p+2)$ and all $2 \leq p<+\infty$, and $\left(\Gamma_{\alpha \beta}^{\rho}\right)^{n} \partial_{\rho} u^{n} \cdot a_{3}^{n} \rightarrow \Gamma_{\alpha \beta}^{\rho} \partial_{\rho} u \cdot a_{3}$ weakly in $L^{2}(\omega)$. Since the embedding $L^{2}(\omega) \hookrightarrow H^{-1}(\omega)$ is compact, it follows that $\left(\Gamma_{\alpha \beta}^{\rho}\right)^{n} \partial_{\rho} u^{n} \cdot a_{3}^{n} \rightarrow \Gamma_{\alpha \beta}^{\rho} \partial_{\rho} u \cdot a_{3}$ strongly in $H^{-1}(\omega)$.

Let us now consider the other term. We have

$$
\left\|\partial_{\alpha \beta} u^{n} \cdot a_{3}^{n}-\partial_{\alpha \beta} u \cdot a_{3}\right\|_{H^{-1}(\omega)}=\sup _{\|\theta\|_{H_{0}^{1}(\omega)}=1}\left|\int_{\omega}\left[\partial_{\alpha} u^{n} \cdot \partial_{\beta}\left(\theta a_{3}^{n}\right)-\partial_{\alpha} u \cdot \partial_{\beta}\left(\theta a_{3}\right)\right] d x\right|
$$

by formula (21). Let us estimate the right-hand side of this equality:

$$
\begin{aligned}
\left\|\partial_{\alpha \beta} u^{n} \cdot a_{3}^{n}-\partial_{\alpha \beta} u \cdot a_{3}\right\|_{H^{-1}(\omega)} \leq \sup _{\|\theta\|_{H_{0}^{1}(\omega)}=1}\left|\int_{\omega} \partial_{\alpha}\left(u^{n}-u\right) \cdot \partial_{\beta}\left(\theta a_{3}^{n}\right) d x\right| \\
\quad+\sup _{\|\theta\|_{H_{0}^{1}(\omega)}^{1}=1}\left|\int_{\omega} \partial_{\beta} \theta \partial_{\alpha} u \cdot\left(a_{3}^{n}-a_{3}\right) d x\right| \\
+\sup _{\|\theta\|_{H_{0}^{1}(\omega)=1}}\left|\int_{\omega} \theta \partial_{\alpha} u \cdot \partial_{\beta}\left(a_{3}^{n}-a_{3}\right) d x\right| .
\end{aligned}
$$

The first two terms of (22) clearly tend to 0 as $n \rightarrow+\infty$. Let us examine the last term in more detail. Note first that $a_{3}^{n} \rightarrow a_{3}$ strongly in $W^{1, p}\left(\omega ; \mathbb{R}^{3}\right)$ for all $p>2$ since the space $W^{1, p}(\omega)$ is a Banach algebra. By Hölder's inequality, we thus have

$$
\left|\int_{\omega} \theta \partial_{\alpha} u \cdot \partial_{\beta}\left(a_{3}^{n}-a_{3}\right) d x\right| \leq\left\|\partial_{\alpha} u\right\|_{L^{2}\left(\omega ; \mathbb{R}^{3}\right)}\|\theta\|_{L^{\frac{2 p}{p-2}(\omega)}}\left\|\partial_{\beta}\left(a_{3}^{n}-a_{3}\right)\right\|_{L^{p}\left(\omega ; \mathbb{R}^{3}\right)} .
$$

By the Sobolev embedding theorem, $\|\theta\|_{L^{\frac{2 p}{p-2}(\omega)}} \leq C_{p}\|\theta\|_{H_{0}^{1}(\omega)}$ and thus

$$
\sup _{\|\theta\|_{H_{0}^{1}(\omega)}=1}\left|\int_{\omega} \theta \partial_{\alpha} u \cdot \partial_{\beta}\left(a_{3}^{n}-a_{3}\right) d x\right| \rightarrow 0 \quad \text { as } n \rightarrow+\infty .
$$

Combining together the two convergences thus established, we see that $\Upsilon_{\alpha \beta}^{\text {new }}\left(u^{n}\right) \rightarrow$ $\Upsilon_{\alpha \beta}^{\text {new }}(u)$ strongly in $H^{-1}(\omega)$. This holds true in particular when $u^{n}$ and $\varphi^{n}$ are regular, which completes the proof.

REMARK. In the above proof, we could have assumed just as well that $\varphi \in W^{2, p}(\omega$; $\left.\mathbb{R}^{3}\right)$ for some $p>2$ instead of $\varphi \in W^{2, \infty}\left(\omega ; \mathbb{R}^{3}\right)$.

\section{A new functional setting for the infinitesimal rigid displacement lemma.} In what follows, the midsurface is always assumed to be such that $\varphi \in W^{2, \infty}\left(\omega ; \mathbb{R}^{3}\right)$ unless otherwise specified. The purpose of this section is twofold. First, we introduce a new functional framework for Koiter's model and prove that it provides a natural extension of the classical framework of Bernadou and Ciarlet [2]. Second, we establish 
the infinitesimal displacement lemma in this functional framework. The infinitesimal displacement lemma is a key ingredient in the existence and uniqueness proof of Sec. 5 below.

Let us introduce the space

$$
V=\left\{v \in H_{0}^{1}\left(\omega ; \mathbb{R}^{3}\right), \quad \partial_{\alpha \beta} v \cdot a_{3} \in L^{2}(\omega)\right\}
$$

which we equip with the norm

$$
\|v\|_{V}=\left(\|v\|_{H^{1}\left(\omega ; \mathbb{R}^{3}\right)}^{2}+\sum_{\alpha, \beta}\left\|\partial_{\alpha \beta} v \cdot a_{3}\right\|_{L^{2}(\omega)}^{2}\right)^{1 / 2} .
$$

We recall that, if $v \in H^{1}\left(\omega ; \mathbb{R}^{3}\right)$ then $\partial_{\alpha \beta} v \cdot a_{3}$ is a distribution of $H^{-1}(\omega)$ defined by

$$
\forall \theta \in H_{0}^{1}(\omega), \quad\left\langle\partial_{\alpha \beta} v \cdot a_{3}, \theta\right\rangle=-\left\langle\partial_{\alpha} v_{i}, \partial_{\beta}\left(\left(a_{3}\right)_{i} \theta\right)\right\rangle,
$$

where the components are the Cartesian components. Hence the space $V$ is well defined as a subspace of $H^{1}\left(\omega ; \mathbb{R}^{3}\right)$.

Lemma 3. The space $V$ is a Hilbert space.

Proof. Clear.

Note that elements $v$ of $V$ are such that $\gamma_{\alpha \beta}(v) \in L^{2}(\omega)$ and $\Upsilon_{\alpha \beta}(v) \in L^{2}(\omega)$ by expressions (11) and (12).

Let us now prove that the space $V$ defines a natural extension of the classical functional framework of Bernadou and Ciarlet [2] to our case.

Lemma 4. Assume that $\varphi \in W^{3, \infty}\left(\omega ; \mathbb{R}^{3}\right)$. Then the space $V$ is equal to the space of displacements $v=v_{i} a^{i} \in H_{0}^{1}\left(\omega ; \mathbb{R}^{3}\right)$ whose covariant components $\left(v_{i}\right)_{i=1,2,3}$ belong to the space $V_{0}=H_{0}^{1}(\omega) \times H_{0}^{1}(\omega) \times\left(H^{2}(\omega) \cap H_{0}^{1}(\omega)\right)$. When $V_{0}$ is equipped with the norm

$$
\left\|\left(v_{i}\right)\right\|_{V_{0}}=\left(\sum_{\alpha}\left\|v_{\alpha}\right\|_{H^{1}(\omega)}^{2}+\left\|v_{3}\right\|_{H^{2}(\omega)}^{2}\right)^{2},
$$

this correspondence defines an isomorphism.

Proof. Let $v$ be an element of $V$. First of all, the covariant components $v_{i}=v \cdot a_{i}$ belong to $L^{2}(\omega)$ since $v \in L^{2}\left(\omega ; \mathbb{R}^{3}\right)$ and $a_{i} \in L^{\infty}\left(\omega ; \mathbb{R}^{3}\right)$. Secondly, $\partial_{\alpha} v_{i}=\partial_{\alpha} v \cdot a_{i}+$ $v \cdot \partial_{\alpha} a_{i} \in L^{2}(\omega)$ since $v \in H^{1}\left(\omega ; \mathbb{R}^{3}\right)$ and $a_{i} \in W^{1, \infty}\left(\omega ; \mathbb{R}^{3}\right)$, so that $v_{i} \in H^{1}(\omega)$. Moreover, it is clear that the trace of $v_{i}$ on $\partial \omega$ is zero, and hence $v_{i} \in H_{0}^{1}(\omega)$. Finally, $\partial_{\alpha \beta} v_{3}=\partial_{\alpha \beta} v \cdot a_{3}+\partial_{\alpha} v \cdot \partial_{\beta} a_{3}+\partial_{\alpha} a_{3} \cdot \partial_{\beta} v+\partial_{\alpha \beta} a_{3} \cdot v \in L^{2}(\omega)$ since $a_{3} \in W^{2, \infty}\left(\omega ; \mathbb{R}^{3}\right)$. Therefore $\left(v_{i}\right)$ is in $V_{0}$.

Conversely, let $\left(v_{1}, v_{2}, v_{3}\right)$ be an element of $V_{0}$ and let $v=v_{i} a^{i} \in L^{2}\left(\omega ; \mathbb{R}^{3}\right)$. Then

$$
\partial_{\alpha} v=\left(\partial_{\alpha} v_{\mu}-\Gamma_{\alpha \mu}^{\rho} v_{\rho}-b_{\alpha \mu} v_{3}\right) a^{\mu}+\left(\partial_{\alpha} v_{3}+b_{\alpha}^{\mu} v_{\mu}\right) a^{3} \in L^{2}\left(\omega ; \mathbb{R}^{3}\right)
$$

so that $v \in H^{1}\left(\omega ; \mathbb{R}^{3}\right)$. Moreover, it follows from (28) that

$$
\begin{gathered}
\partial_{\alpha \beta} v=\partial_{\beta}\left[\partial_{\alpha} v_{\mu}-\Gamma_{\alpha \mu}^{\rho} v_{\rho}-b_{\alpha \mu} v_{3}\right] a^{\mu}+\left[\partial_{\alpha} v_{\mu}-\Gamma_{\alpha \mu}^{\rho} v_{\rho}-b_{\alpha \mu} v_{3}\right] \partial_{\beta} a^{\mu} \\
+\partial_{\beta}\left[\partial_{\alpha} v_{3}+b_{\alpha}^{\mu} v_{\mu}\right] a^{3}+\left[\partial_{\alpha} v_{3}+b_{\alpha}^{\mu} v_{\mu}\right] \partial_{\beta} a^{3}
\end{gathered}
$$


as an element of $H^{-1}\left(\omega ; \mathbb{R}^{3}\right)$. We deduce from $(29)$ that

$$
\partial_{\alpha \beta} v \cdot a_{3}=\left[\partial_{\alpha} v_{\mu}-\Gamma_{\alpha \mu}^{\rho} v_{\rho}-b_{\alpha \mu} v_{3}\right] b_{\beta}^{\mu}+\partial_{\alpha \beta} v_{3}+\partial_{\beta}\left[b_{\alpha}^{\mu} v_{\mu}\right] \in L^{2}(\omega) .
$$

Finally, since the vectors $a^{i}$ belong to $W^{2, \infty}\left(\omega ; \mathbb{R}^{3}\right)$ by assumption and $v_{i} \in H_{0}^{1}(\omega)$, it follows that $v=v_{i} a^{i} \in H_{0}^{1}\left(\omega ; \mathbb{R}^{3}\right)$. We conclude that $V=V_{0}$ algebraically.

It follows from formula (30) that for all $v \in V_{0}$

$$
\left\|\partial_{\alpha \beta} v \cdot a_{3}\right\|_{L^{2}(\omega)} \leq C\left(\left\|\partial_{\alpha \beta} v_{3}\right\|_{L^{2}(\omega)}+\sum_{\alpha, \beta}\left\|\partial_{\alpha} v_{\beta}\right\|_{L^{2}(\omega)}+\sum_{i}\left\|v_{i}\right\|_{L^{2}(\omega)}\right)
$$

since $\Gamma_{\alpha \beta}^{\rho}, b_{\alpha \beta}$, and $b_{\alpha}^{\beta}$ belong to $W^{1, \propto}(\omega)$. Similarly, formula (28) implies that

$$
\left\|\partial_{\alpha} v\right\|_{L^{2}\left(\omega ; \mathbb{R}^{3}\right)} \leq C\left(\sum_{i}\left\|\partial_{\alpha} v_{i}\right\|_{L^{2}(\omega)}+\sum_{i}\left\|v_{i}\right\|_{L^{2}(\omega)}\right)
$$

for the same reason. Finally, it is clear that

$$
\|v\|_{L^{2}\left(\omega ; \mathbb{R}^{3}\right)} \leq C\left(\sum_{i}\left\|v_{i}\right\|_{L^{2}(\omega)}\right) .
$$

We infer from the last three estimates that for all $v \in V_{0},\|v\|_{V} \leq C\|v\|_{V_{0}}$. Hence the embedding $V_{0} \hookrightarrow V$ is continuous and so is its inverse by the open mapping theorem. Therefore $V$ and $V_{0}$ are isomorphic.

REMARK. The space $V_{0}$ was introduced in the classical approach of Bernadou and Ciarlet [2]; see also Bernadou, Ciarlet and Miara [3] when the midsurface is regular, for instance $\varphi \in C^{3}\left(\omega ; \mathbb{R}^{3}\right)$.

Corollary 5. Assume that $\varphi \in W^{3, \infty}\left(\omega ; \mathbb{R}^{3}\right)$ and $v \in V$. Then the classical expressions for the linearized strain and change of curvature tensors and the new expressions (11) and (12) coincide.

Proof. This follows directly from Lemmas 2 and 4.

Let us turn to the infinitesimal rigid displacement lemma in our functional framework.

Theorem 6. Assume that $\varphi \in W^{2, \infty}\left(\omega ; \mathbb{R}^{3}\right)$. Let $u \in H^{1}\left(\omega ; \mathbb{R}^{3}\right)$ be a displacement of the surface $S$.

i) If $u$ satisfies $\gamma(u)=0$ then there exists a unique $\psi \in L^{2}\left(\omega ; \mathbb{R}^{3}\right)$ such that

$$
\partial_{\alpha} u=\psi \wedge \partial_{\alpha} \varphi, \quad \alpha=1,2 .
$$

ii) (Infinitesimal rigid displacement lemma) If in addition $\Upsilon(u)=0$ then $\psi$ is identified with a constant vector of $\mathbb{R}^{3}$ and we have for all $x \in \omega$

$$
u(x)=c+\psi \wedge \varphi(x)
$$

where $c \in \mathbb{R}^{3}$ is a constant vector.

REMARKS. i) Theorem 6 contains the infinitesimal rigid displacement lemma of Bernadou and Ciarlet [2], see also Bernadou, Ciarlet and Miara [3], under weaker hypotheses of regularity for the midsurface and the displacement. See Blouza and Le Dret [5] for other 
versions of this result under various hypotheses of regularity. Note that if $u \in H^{1}\left(\omega ; \mathbb{R}^{3}\right)$ satisfies $\gamma(u)=0$ then $u \cdot a_{\alpha} \in H^{2}(\omega)$ and $\psi \cdot a^{3} \in H^{1}(\omega)$ (see below).

ii) The vector field $\psi$ is called the infinitesimal rotation field. It is given in the classical case by the relation

$$
\psi=\psi(u)=\epsilon^{\alpha \beta}\left(\partial_{\beta} u_{3}+b_{\beta}^{\rho} u_{\rho}\right) a_{\alpha}+\frac{1}{2} \epsilon^{\alpha \beta} u_{\alpha \mid \beta} a_{3}
$$

where $\epsilon^{11}=\epsilon^{22}=0$ and $\epsilon^{12}=-\epsilon^{21}=1 / \sqrt{a}$; see Vekua [21], Bernadou and Ciarlet [2], Choi and Sanchez-Palencia [8] and Choi [7].

We divide the proof of Theorem 6 into three steps. The first step consists in extending some elementary results of vector calculus to a distributional framework. For the duration of this step, all components are Cartesian components. Recall that

$$
\epsilon_{i j k}= \begin{cases}1 & \text { if }\{i, j, k\} \text { is an even permutation of }\{1,2,3\} \\ -1 & \text { if }\{i, j, k\} \text { is an odd permutation of }\{1,2,3\} \\ 0 & \text { otherwise }\end{cases}
$$

LEMma 7. Let $v \in H^{-1}\left(\omega ; \mathbb{R}^{3}\right)$ (resp. $\left.L^{2}\left(\omega ; \mathbb{R}^{3}\right)\right)$ and let $a \in W^{1, \infty}\left(\omega ; \mathbb{R}^{3}\right)$ be such that $|a(x)| \geq \delta>0$ on $\bar{\omega}$.

i) If $v \cdot a=0$, i.e., for every $\theta \in H_{0}^{1}(\omega),\langle v \cdot a, \theta\rangle=\left\langle v_{i}, a_{i} \theta\right\rangle=0$ (resp. a.e. in $\omega$ ) then there exists $w \in H^{-1}\left(\omega ; \mathbb{R}^{3}\right)$ (resp. $\left.L^{2}\left(\omega ; \mathbb{R}^{3}\right)\right)$ such that $v=w \wedge a$, i.e., for every $\theta \in H_{0}^{1}(\omega),\left\langle v_{i}, \theta\right\rangle=\epsilon_{i j k}\left\langle w_{j}, a_{k} \theta\right\rangle$.

ii) If $v \wedge a=0$, i.e., for all $\theta \in H_{0}^{1}(\omega), \epsilon_{i j k}\left\langle v_{j}, a_{k} \theta\right\rangle=0$ (resp. a.e. in $\omega$ ), then there exists $\bar{v} \in H^{-1}(\omega)$ (resp. $\left.L^{2}(\omega)\right)$ such that $v=\bar{v} a$, i.e., for all $\theta \in H_{0}^{1}(\omega),\left\langle v_{i}, \theta\right\rangle=\left\langle\bar{v}, a_{i} \theta\right\rangle$ (resp. a.e. in $\omega)$.

Proof. i) Let $v \in H^{-1}\left(\omega ; \mathbb{R}^{3}\right)$ and $w=\frac{a \wedge v}{|a|^{2}}$. Then $w$ is a distribution of $H^{-1}\left(\omega ; \mathbb{R}^{3}\right)$ defined by

$$
\forall \theta \in H_{0}^{1}(\omega), \quad\left\langle w_{i}, \theta\right\rangle=\epsilon_{i j k}\left\langle v_{k}, \frac{a_{j}}{|a|^{2}} \theta\right\rangle .
$$

Indeed, $a_{j} \theta /|a|^{2}$ is an element of $H_{0}^{1}(\omega)$ since $a_{j} \in W^{1, \infty}\left(\omega ; \mathbb{R}^{3}\right)$ and, as $1 /|a|^{2} \leq 1 / \delta^{2}$, $1 /|a|^{2} \in W^{1, \infty}\left(\omega ; \mathbb{R}^{3}\right)$ too. Then we have for all $\theta \in H_{0}^{1}(\omega)$,

$$
\left\langle(w \wedge a)_{i}, \theta\right\rangle=\epsilon_{i j k} \epsilon_{j m n}\left\langle v_{n}, \frac{a_{m} a_{k}}{|a|^{2}} \theta\right\rangle=-\left\langle v_{k}, \frac{a_{k} a_{i}}{|a|^{2}} \theta\right\rangle+\left\langle v_{i}, \frac{a_{k} a_{k}}{|a|^{2}} \theta\right\rangle=\left\langle v_{i}, \theta\right\rangle,
$$

since

$$
\epsilon_{i j k} \epsilon_{j m n}= \begin{cases}1 & \text { if }(i, k)=(n, m) \\ -1 & \text { if }(i, k)=(m, n) \\ 0 & \text { otherwise. }\end{cases}
$$

ii) Let $v \in H^{-1}\left(\omega ; \mathbb{R}^{3}\right)$ and set $\bar{v}=v \cdot a /|a|^{2}$. Then $\bar{v}$ is an element of $H^{-1}(\omega)$ defined by $\langle\bar{v}, \theta\rangle=\left\langle v_{j}, \frac{a_{j}}{|a|^{2}} \theta\right\rangle$ for all $\theta \in H_{0}^{1}(\omega)$. Indeed, $\frac{a_{j}}{|a|^{2}}$ belongs to $W^{1, \infty}\left(\omega, \mathbb{R}^{3}\right)$ and therefore $\frac{a_{j}}{|a|^{2}} \theta$ is an element of $H_{0}^{1}(\omega)$. Let us check that $\left\langle\bar{v}, a_{i} \theta\right\rangle=\left\langle v_{i}, \theta\right\rangle$ for $i=1$. We have

$$
\left\langle\bar{v}, a_{1} \theta\right\rangle=\left\langle v_{1}, \frac{a_{1} a_{1}}{|a|^{2}} \theta\right\rangle+\left\langle v_{2}, \frac{a_{2} a_{1}}{|a|^{2}} \theta\right\rangle+\left\langle v_{3}, \frac{a_{3} a_{1}}{|a|^{2}} \theta\right\rangle .
$$


Now, since by assumption $v \wedge a=0$, i.e., $\epsilon_{i j k}\left(v_{k}, a_{j} \theta\right)=0$, it follows that

$$
\left\langle\bar{v}, a_{1} \theta\right\rangle=\left\langle v_{1}, \frac{a_{1}^{2}}{|a|^{2}} \theta\right\rangle+\left\langle v_{1}, \frac{a_{2}^{2}}{|a|^{2}} \theta\right\rangle+\left\langle v_{1}, \frac{a_{3}^{2}}{|a|^{2}} \theta\right\rangle=\left\langle v_{1}, \theta\right\rangle .
$$

We repeat the argument for $i=2$ and $i=3$.

To conclude the proof, we remark that if $v \in L^{2}\left(\omega ; \mathbb{R}^{3}\right)$ then the above construction gives $w \in L^{2}\left(\omega ; \mathbb{R}^{3}\right)$ and $\bar{v} \in L^{2}(\omega)$ and the equalities hold almost everywhere in $\omega$ and not just in the distributional sense.

We now are in a position to prove the existence of the infinitesimal rotation vector for inextensional displacements, i.e., displacements whose strain tensor vanishes.

Lemma 8. Let $u \in H^{1}\left(\omega ; \mathbb{R}^{3}\right)$ and $\varphi \in W^{1, \infty}\left(\omega ; \mathbb{R}^{3}\right)$ be such that $\gamma(u)=0$ a.e. in $\omega$. Then there exists $\psi \in L^{2}\left(\omega ; \mathbb{R}^{3}\right)$ such that (34) holds.

REMARK. Note that for this result, $\varphi$ is only in $W^{1, \infty}\left(\omega ; \mathbb{R}^{3}\right)$.

Proof. According to expression (11), we see that

$$
\left\{\begin{array}{l}
\partial_{\alpha} u \cdot a_{\alpha}=0 \text { a.e. in } \omega \text { (without summation), } \\
\partial_{1} u \cdot a_{2}+\partial_{2} u \cdot a_{1}=0 \text { a.e. in } \omega .
\end{array}\right.
$$

By the first two equations and by Lemma $7 \mathrm{i}$ ), we know that there exist $w_{1}$ and $w_{2}$ in $L^{2}\left(\omega ; \mathbb{R}^{3}\right)$ such that $\partial_{\alpha} u=w_{\alpha} \wedge a_{\alpha}$ a.e. in $\omega$ (without summation).

In addition, the third equation implies that $\left(w_{1} \wedge a_{1}\right) \cdot a_{2}+\left(w_{2} \wedge a_{2}\right) \cdot a_{1}=0$ or equivalently $\left(w_{1}-w_{2}\right) \cdot a_{3}=0$ a.e. in $\omega$. Consequently, $w_{1}-w_{2}=\left(\left(w_{1}-w_{2}\right) \cdot a^{i}\right) a_{i}=\left(\left(w_{1}-\right.\right.$ $\left.\left.w_{2}\right) \cdot a^{\alpha}\right) a_{\alpha}$ (we recall that $\left.a_{3}=a^{3}\right)$. Let $\psi=w_{1}-\left(\left(w_{1}-w_{2}\right) \cdot a^{1}\right) a_{1}=w_{2}+\left(\left(w_{1}-w_{2}\right) \cdot a^{2}\right) a_{2}$ a.e. in $\omega$. It is clear that

$$
\left\{\begin{array}{l}
\psi \wedge a_{1}=w_{1} \wedge a_{1}=\partial_{1} u \text { a.e. in } \omega \\
\psi \wedge a_{2}=w_{2} \wedge a_{2}=\partial_{2} u \text { a.e. in } \omega
\end{array}\right.
$$

which proves the existence of the infinitesimal rotation vector.

Concerning the uniqueness, we remark that if $\psi^{\prime} \in L^{2}\left(\omega ; \mathbb{R}^{3}\right)$ is such that $\psi^{\prime} \wedge a_{\alpha}=0$ a.e. in $\omega$, the $\psi^{\prime}=0$ a.e. in $\omega$. Indeed, Lemma 7ii) implies that there exist $\bar{\psi}_{1}^{\prime}$ and $\bar{\psi}_{2}^{\prime}$ in $L^{2}(\omega)$ such that $\psi^{\prime}=\psi_{1}^{\prime} a_{1}=\psi_{2}^{\prime} a_{2}$. Multiplying this equality by $a^{1}$, we obtain that $\psi_{2}^{\prime}=0$ a.e. in $\omega$, and hence $\psi^{\prime}=0$.

REMARKs. i) If $\varphi \in W^{2, \infty}\left(\omega ; \mathbb{R}^{3}\right)$ and $\gamma(u)=0$, then we have $\partial_{\alpha}\left(u \cdot a_{\alpha}\right)=u \cdot \partial_{\alpha} a_{\alpha} \in$ $H^{1}(\omega)$ (without summation) and $\partial_{1}\left(u \cdot a_{2}\right)+\partial_{2}\left(u \cdot a_{1}\right)=u \cdot \partial_{1} a_{2}+u \cdot \partial_{2} a_{1} \in H^{1}(\omega)$. By the two-dimensional Korn inequality we immediately infer that $u_{\alpha}=u \cdot a_{\alpha} \in H^{2}(\omega)$; see Geymonat and Sanchez-Palencia [15] for similar observations.

ii) In this case, if we multiply the first line of (39) by $a_{2}$ and the second line by $a_{1}$, we obtain that $\partial_{1} u \cdot a_{2}=\left|a_{1} \wedge a_{2}\right|\left(a_{3} \cdot \psi\right)$ and $\partial_{2} u \cdot a_{1}=-\left|a_{1} \wedge a_{2}\right|\left(a_{3} \cdot \psi\right)$. It thus follows that $2\left|a_{1} \wedge a_{2}\right|\left(a_{3} \cdot \psi\right)=\partial_{1} u \cdot a_{2}-\partial_{2} u \cdot a_{1}=\partial_{1}\left(u \cdot a_{2}\right)-\partial_{2}\left(u \cdot a_{1}\right) \in H^{1}(\omega)$ since $\partial_{2} a_{1}=\partial_{1} a_{2}$. This shows that $\psi_{3}=\psi \cdot a_{3} \in H^{1}(\omega)$.

We now conclude the proof of Theorem 6 .

Lemma 9. Let $u \in H^{1}\left(\omega ; \mathbb{R}^{3}\right)$ and $\varphi \in W^{2, \infty}\left(\omega ; \mathbb{R}^{3}\right)$. If $u$ satisfies $\gamma(u)=0$ and $\Upsilon(u)=$ 0 , then $\psi$ is a constant vector and we have $u(x)=c+\psi \wedge \varphi(x)$ where $c \in \mathbb{R}^{3}$ is a constant vector. 
Proof. Since $u$ is in $H^{1}\left(\omega ; \mathbb{R}^{3}\right)$ then $\partial_{\alpha \beta} u$ is a distribution of $H^{-1}\left(\omega ; \mathbb{R}^{3}\right)$. In fact, because of (34), we have

$$
\partial_{\alpha, \beta} u=\partial_{\beta} \psi \wedge a_{\alpha}+\psi \wedge \partial_{\beta} a_{\alpha}
$$

Indeed, for all $\theta \in H_{0}^{1}\left(\omega ; \mathbb{R}^{3}\right), a_{\alpha} \wedge \theta \in H_{0}^{1}\left(\omega ; \mathbb{R}^{3}\right)$ and

$$
\left\langle\partial_{\beta} \psi \wedge a_{\alpha}, \theta\right\rangle=\left\langle\partial_{\beta} \psi, a_{\alpha} \wedge \theta\right\rangle=-\int_{\omega} \partial_{\beta}\left(a_{\alpha} \wedge \theta\right) \cdot \psi d x
$$

and since $\partial_{\beta}\left(a_{\alpha} \wedge \theta\right)=\partial_{\beta} a_{\alpha} \wedge \theta+a_{\alpha} \wedge \partial_{\beta} \theta$, it follows from (41) that

$$
\begin{aligned}
\left\langle\partial_{\beta} \psi \wedge a_{\alpha}, \theta\right\rangle & =-\int_{\omega} \partial_{\beta} a_{\alpha} \wedge \theta \cdot \psi d x-\int_{\omega} a_{\alpha} \wedge \partial_{\beta} \theta \cdot \psi d x \\
& =-\int_{\omega} \psi \wedge \partial_{\beta} a_{\alpha} \cdot \theta d x-\int_{\omega} \psi \wedge a_{\alpha} \cdot \partial_{\beta} \theta d x \\
& =-\left\langle\psi \wedge \partial_{\beta} a_{\alpha}, \theta\right\rangle+\left\langle\partial_{\beta}\left(\psi \wedge a_{\alpha}\right), \theta\right\rangle,
\end{aligned}
$$

which proves (40).

The distribution $\partial_{\alpha \beta} u \cdot a_{3} \in H^{-1}(\omega)$ thus satisfies

$$
\begin{aligned}
\partial_{\alpha \beta} u \cdot a_{3} & =\left(\partial_{\beta} \psi \wedge a_{\alpha}\right) \cdot a_{3}+\left(\psi \wedge \partial_{\beta} a_{\alpha}\right) \cdot a_{3} \\
& =\left(\partial_{\beta} \psi \wedge a_{\alpha}\right) \cdot a_{3}+\Gamma_{\alpha \beta}^{\rho}\left(\psi \wedge a_{\rho}\right) \cdot a_{3}+b_{\alpha \beta}\left(\psi \wedge a_{3}\right) \cdot a_{3} \\
& =\left(\partial_{\beta} \psi \wedge a_{\alpha}\right) \cdot a_{3}+\Gamma_{\alpha \beta}^{\rho} \partial_{\rho} u \cdot a_{3} .
\end{aligned}
$$

Note that the last two terms in $(43)_{2}$ belong to $L^{2}(\omega)$.

Consequently $\Upsilon(u)=0$ implies that $\left(\partial_{\beta} \psi \wedge a_{\alpha}\right) \cdot a_{3}=\left(a_{3} \wedge \partial_{\beta} \psi\right) \cdot a_{\alpha}=0$. In addition, we have $\left(a_{3} \wedge \partial_{\beta} \psi\right) \cdot a_{3}=0$ by an immediate density argument. In Cartesian coordinates, these statements read

$$
\forall \theta \in H_{0}^{1}(\omega), \quad\left\langle\left(a_{3} \wedge \partial_{\beta} \psi\right)_{k},\left(a_{i}\right)_{k} \theta\right\rangle=0, \quad i=1,2,3 .
$$

Consider now an arbitrary function $\phi \in \mathcal{D}\left(\omega ; \mathbb{R}^{3}\right)$. Due to the regularity of the chart $\varphi$, the contravariant components of $\phi, \phi^{i}=\phi \cdot a^{i}$, belong to $H_{0}^{1}(\omega)$ and $\phi=\phi^{i} a_{i}$. Using $\phi^{i}$ as a test function in (44) we thus obtain

$$
\forall \phi \in \mathcal{D}\left(\omega ; \mathbb{R}^{3}\right), \quad\left\langle\left(a_{3} \wedge \partial_{\beta} \psi\right)_{k}, \phi^{i}\left(a_{i}\right)_{k}\right\rangle=\left\langle a_{3} \wedge \partial_{\beta} \psi, \phi\right\rangle=0 ;
$$

hence

$$
a_{3} \wedge \partial_{\beta} \psi=0 \text {. }
$$

Thanks to (46) and Lemma 7 , there exist $\bar{\psi}_{1}^{\prime}, \bar{\psi}_{2}^{\prime} \in H^{-1}(\omega)$ such that $\partial_{\beta} \psi=\bar{\psi}_{\beta}^{\prime} a_{3}$. Since $\partial_{\alpha \beta} u=\partial_{\beta \alpha} u$, it follows from formula (40) that $\partial_{2} \psi \wedge a_{1}=\partial_{1} \psi \wedge a_{2}$; in other words $\bar{\psi}_{1}^{\prime}\left(a_{3} \wedge a_{1}\right)=\bar{\psi}_{2}^{\prime}\left(a_{3} \wedge a_{2}\right)$. Therefore we have $\bar{\psi}_{1}^{\prime}=\bar{\psi}_{2}^{\prime}=0$ and thus $\partial_{\beta} \psi=0$. Since $\omega$ is a domain, this implies that $\psi$ is identified with a constant vector of $\mathbb{R}^{3}$. To conclude we remark that then $\partial_{\beta}(u-\psi \wedge \varphi)=0$; hence $u-\psi \wedge \varphi$ is identified with a constant vector $c$ of $\mathbb{R}^{3}$.

See Blouza and Le Dret [5] for similar results under various hypotheses of regularity. 
5. Existence and uniqueness for Koiter's model. In this section, we propose to prove the existence and uniqueness of the solution of the linearized Koiter model for a shell whose midsurface may have curvature discontinuities, since $\varphi$ is only in $W^{2, \infty}\left(\omega ; \mathbb{R}^{3}\right)$. To begin with we consider the case of simple support on the entire boundary. We will consider more general boundary conditions in the next section.

Let $a^{\alpha \beta \rho \sigma} \in L^{\infty}(\omega)$ be an elasticity tensor that we assume to satisfy the usual symmetries and to be uniformly strictly positive, i.e., there exists a constant $C>0$ such that for all symmetric tensors $\tau=\left(\tau_{\alpha \beta}\right)$ and almost all $x \in \omega, a^{\alpha \beta \rho \sigma}(x) \tau_{\alpha \beta} \tau_{\rho \sigma} \geq C\left(\tau_{\alpha \beta} \tau_{\alpha \beta}\right)$. These hypotheses are for example satisfied by a homogeneous, isotropic material with Lamé moduli $\mu>0$ and $\lambda \geq 0$, in which case

$$
a^{\alpha \beta \rho \sigma}=2 \mu\left(a^{\alpha \rho} a^{\beta \sigma}+a^{\alpha \sigma} a^{\beta \rho}\right)+\frac{4 \lambda \mu}{\lambda+2 \mu} a^{\alpha \beta} a^{\rho \sigma} .
$$

Finally let $e \in L^{\infty}(\omega)$, such that $e(x) \geq C>0$ almost everywhere on $\omega$, represent the thickness of the shell. This means that the actual three-dimensional shell is the set of points $M=\varphi(x)+z a_{3}(x)$ with $x \in \omega$ and $|z|<e(x)$.

Theorem 10. Let $\varphi \in W^{2, \infty}\left(\omega ; \mathbb{R}^{3}\right)$ and let $P \in L^{2}\left(\omega ; \mathbb{R}^{3}\right)$ be a given force resultant density. Then there exists a unique solution to the variational problem: Find $u \in V$ such that

$$
\forall v \in V, \quad \int_{\omega} e a^{\alpha \beta \rho \sigma}\left(\gamma_{\alpha \beta}(u) \gamma_{\rho \sigma}(v)+\frac{e^{2}}{12} \Upsilon_{\alpha \beta}(u) \Upsilon_{\rho \sigma}(v)\right) \sqrt{a} d x=\int_{\omega} P \cdot v \sqrt{a} d x
$$

REMARK. In view of Lemma 4 and Corollary 5 , if $\varphi$ is assumed to be of class $C^{3}$, we thus recover the result of Bernadou and Ciarlet [2].

Note first that the right-hand side of problem (47) clearly defines a continuous linear form over the space $V$. We thus only need to prove that the bilinear form of the left-hand side of (47) is $V$-elliptic. This is the object of the next lemma.

Lemma 11. The bilinear form of the left-hand side of (47) is $V$-elliptic.

Proof. Under the hypotheses made on the chart $\varphi$, the elasticity tensor and the thickness of the shell, we only need to prove that

$$
\|v\|=\left(\sum_{\alpha, \beta}\left\|\gamma_{\alpha \beta}(v)\right\|_{L^{2}(\omega)}^{2}+\sum_{\alpha, \beta}\left\|\Upsilon_{\alpha \beta}(v)\right\|_{L^{2}(\omega)}^{2}\right)^{1 / 2}
$$

is a norm over the space $V$ that is equivalent to $\|\cdot\|_{V}$.

First of all, let us check that the mapping $v \in V \mapsto\||v|\|$ is indeed a norm over $V$. It is clear that this mapping is a semi-norm. By Theorem 6 , if $v \in V$ is such that $\|\mid v\| \|=0$, then there exist $\psi, c \in \mathbb{R}^{3}$ such that $v(x)=\psi \wedge \varphi(x)+c$. The set of points $y \in \mathbb{R}^{3}$ such that $\psi \wedge y+c$ vanishes is either a straight line $(\psi \neq 0$ and $c \neq 0)$, empty $(\psi=0$ and $c \neq 0)$ or the whole space $(\psi=0$ and $c=0)$. Since $v$ vanishes on $\partial \omega$ and $\varphi(\partial \omega)$ is not included in a straight line, it follows that $v=0$. 
Let us now prove that the norm $\||\cdot|\|$ is equivalent to the $\|\cdot\|_{V}$ norm. We argue by contradiction. Let us assume that there exists a sequence $v_{n}$ in $V$ such that

$$
\left\|v_{n}\right\|_{V}=1 \text { and }\left\|v_{n}\right\| \rightarrow 0 \text { when } n \rightarrow+\infty \text {. }
$$

By extracting a subsequence, still denoted $v_{n}$, we may assume that there exists a $v \in V$ such that $v_{n} \rightarrow v$ weakly in $H^{1}\left(\omega ; \mathbb{R}^{3}\right)$ and $\partial_{\alpha \beta} v_{n} \cdot a_{3} \rightarrow \partial_{\alpha \beta} v \cdot a_{3}$ weakly in $L^{2}(\omega)$. Consequently,

$$
\gamma_{\alpha \beta}\left(v_{n}\right) \rightarrow \gamma_{\alpha \beta}(v) \text { and } \Upsilon_{\alpha \beta}\left(v_{n}\right) \rightarrow \Upsilon_{\alpha \beta}(v) \text { weakly in } L^{2}(\omega)
$$

by expressions (11) and (12). Since hypothesis (49) implies that these tensors converge strongly to zero in $L^{2}(\omega)$ we obtain $v=0$ thanks to Theorem 6 . Rellich's lemma now implies that $v_{n} \rightarrow 0$ strongly in $L^{2}\left(\omega ; \mathbb{R}^{3}\right)$.

Let us introduce the vector $\left(\bar{w}_{n}\right)_{\alpha}=v_{n} \cdot a_{\alpha}$, which is such that $\bar{w}_{n} \rightarrow 0$ strongly in $L^{2}\left(\omega ; \mathbb{R}^{2}\right)$ by the previous remark. Let us define $2 e_{\alpha \beta}(\bar{w})=\partial_{\beta} \bar{w}_{\alpha}+\partial_{\alpha} \bar{w}_{\beta}$. We see that, by expression (11)

$$
e_{\alpha \beta}\left(\bar{w}_{n}\right)=\gamma_{\alpha \beta}\left(v_{n}\right)+\frac{1}{2} v_{n} \cdot\left(\partial_{\beta} a_{\alpha}+\partial_{\alpha} a_{\beta}\right) \rightarrow 0 \text { strongly in } L^{2}(\omega)
$$

since $a_{\alpha} \in W^{1, \infty}\left(\omega ; \mathbb{R}^{3}\right)$. By the two-dimensional Korn inequality, we deduce then that $\bar{w}_{n} \rightarrow 0$ strongly in $H^{1}\left(\omega ; \mathbb{R}^{2}\right)$. Consequently,

$$
\partial_{\rho} v_{n} \cdot a_{\alpha}=\partial_{\rho}\left(\left(\bar{w}_{n}\right)_{\alpha}\right)-v_{n} \cdot \partial_{f} a_{\alpha} \rightarrow 0 \text { strongly in } L^{2}(\omega)
$$

since $\partial_{\rho} a_{\alpha} \in L^{\infty}\left(\omega ; \mathbb{R}^{3}\right)$.

Moreover, as $v_{n} \rightarrow 0$ in $H^{1}\left(\omega ; \mathbb{R}^{3}\right)$, it follows that $\partial_{\rho} v_{n} \cdot a_{3} \rightarrow 0$ in $L^{2}(\omega)$. On the other hand, $\partial_{\beta}\left(\partial_{\rho} v_{n} \cdot a_{3}\right)=\partial_{\beta \rho} v_{n} \cdot a_{3}+\partial_{\rho} v_{n} \cdot \partial_{\beta} a_{3} \rightarrow 0$ in $L^{2}(\omega)$. Indeed, $\partial_{\beta} a_{3} \in L^{\infty}\left(\omega ; \mathbb{R}^{3}\right)$ and we already know that $\partial_{\beta \rho} v_{n} \cdot a_{3} \rightarrow 0$ weakly in $L^{2}(\omega)$. Consequently, $\partial_{\rho} v_{n} \cdot a_{3} \rightarrow 0$ weakly in $H^{1}(\omega)$ and by Rellich's lemma

$$
\partial_{\rho} v_{n} \cdot a_{3} \rightarrow 0 \text { strongly in } L^{2}(\omega)
$$

We deduce from (53) and (49) that

$$
\partial_{\alpha \beta} v_{n} \cdot a_{3}=\Upsilon_{\alpha \beta}\left(v_{n}\right)+\Gamma_{\alpha \beta}^{\rho} \partial_{\rho} v_{n} \cdot a_{3} \rightarrow 0 \text { strongly in } L^{2}(\omega),
$$

since $\Gamma_{\alpha \beta}^{\rho} \in L^{\infty}(\omega)$, on the one hand, and on the other hand that

$$
\partial_{\rho} v_{n}=\left(\partial_{\rho} v_{n} \cdot a_{i}\right) a^{i} \rightarrow 0 \text { strongly in } L^{2}\left(\omega ; \mathbb{R}^{3}\right)
$$

by (52), (53) and since both $a_{i}$ and $a^{i}$ belong to $L^{\infty}\left(\omega ; \mathbb{R}^{3}\right)$. Consequently, $v_{n} \rightarrow 0$ strongly in $H^{1}\left(\omega ; \mathbb{R}^{3}\right)$. Since by $(54), \partial_{\alpha \beta} v_{n} \cdot a_{3} \rightarrow 0$ strongly in $L^{2}(\omega)$, we see that $\left\|v_{n}\right\|_{V} \rightarrow 0$ which contradicts (49) and proves the lemma.

Theorem 10 now follows directly from Lemma 11 by applying the Lax-Milgram Lemma.

6. Existence and uniqueness for general boundary conditions. In Blouza and Le Dret [6], we gave an existence and uniqueness result for the clamped shell problem for shells of class $W^{2, \infty}$, with the restriction that the midsurface be piecewise $W^{3 . \infty}$. The purpose of this section is to remove this unnecessary restriction. Indeed, we prove below an existence and uniqueness theorem for a Koiter shell clamped on part of the boundary and subjected to given forces and moments on the remaining part of the boundary, under the sole hypothesis $\varphi \in W^{2 \cdot x}\left(\omega ; \mathbb{R}^{3}\right)$. This is achieved by reinterpreting the classical 
conditions of clamping and moment loading in the light of the ideas set forth in the previous sections.

We thus assume that the boundary $\partial \omega$ of the chart domain is divided into two parts, a part $\gamma_{0}$ of strictly positive 1-dimensional measure ${ }^{\dagger}$ on which the shell is clamped and a complementary part $\gamma_{1}$ on which the shell is subjected to applied tractions and moments. In the classical approach, the clamping condition reads

$$
v_{i}=\partial_{\nu} v_{3}=0 \text { on } \gamma_{0}
$$

where $\partial_{\nu}$ denotes the normal derivative on the boundary. The loading condition amounts to adding to the right-hand side of the variational problem a term $l_{1}(v)$ of the form

$$
l_{1}(v)=\int_{\gamma_{1}}(N \cdot v+M \cdot \psi(v)) \sqrt{a_{\alpha \beta} \tau_{\alpha} \tau_{\beta}} d \sigma,
$$

where $\tau$ is a unit tangent vector to $\partial \omega, N=N^{i} a_{i}$ is the applied traction density, $M=$ $M^{\alpha} a_{\alpha} \wedge a_{3}=\epsilon_{\beta \alpha} M^{\alpha} a^{\beta}$ the applied moment density and $\psi(v)$ is the infinitesimal rotation vector, which is still defined by

$$
\psi(v)=\epsilon^{\alpha \beta}\left(\partial_{\beta} v_{3}+b_{\beta}^{\rho} v_{\rho}\right) a_{\alpha}+\frac{1}{2} \epsilon^{\alpha \beta} v_{\alpha \mid \beta} a_{3},
$$

even for a non-necessarily inextensional displacement. Naturally in this case, $\partial_{\alpha} v \neq$ $\psi \wedge a_{\alpha}$.

Our goal now is to show that (56) and (57) can be rewritten in a simpler and more intrinsic fashion that makes sense in our functional framework. We let

$$
W=\left\{v \in H^{1}\left(\omega ; \mathbb{R}^{3}\right) \mid \partial_{\alpha \beta} v \cdot a_{3} \in L^{2}(\omega)\right\},
$$

endowed with the same norm as $V$, which is thus a closed subspace of $W$.

Let us begin with the clamping condition.

Lemma 12. Assume that $\varphi \in W^{2, \infty}\left(\omega ; \mathbb{R}^{3}\right)$ and let $v \in W$ be such that $v=0$ in the sense of trace on $\gamma_{0}$. Then $\partial_{\alpha} v \cdot a_{3} \in H^{1}(\omega)$ with $\left\|\partial_{\alpha} v \cdot a_{3}\right\|_{H^{1}(\omega)} \leq C\|v\|_{W}$ and the condition

$$
\partial_{\alpha} v \cdot a_{3}=0 \text { in } H^{1 / 2}\left(\gamma_{0}\right)
$$

is well defined. Moreover, it is equivalent to (56) if $\varphi \in W^{3, \infty}\left(\omega ; \mathbb{R}^{3}\right)$.

Proof. We first remark that $\partial_{\alpha} v \cdot a_{3} \in H^{1}(\omega)$ with the norm estimate. Indeed,

$$
\partial_{\beta}\left(\partial_{\alpha} v \cdot a_{3}\right)=\partial_{\alpha \beta} v \cdot a_{3}+\partial_{\alpha} v \cdot \partial_{\beta} a_{3} \in L^{2}(\omega) .
$$

Note that the left-hand side of this equality is a priori only in $H^{-1}(\omega)$. The fact that Leibniz' rule holds true in this case can be checked by reasoning along the same lines as in the proof of Lemma 9.

Consequently, condition (60) is well defined for elements of the space $W$. Let us verify that, together with the nullity of the displacement on $\gamma_{0}$, it coincides with the classical clamping condition for $\varphi$ smooth. We let $\nu$ denote the normal outer unit vector to $\partial \omega$ and $\tau$ a tangent unit vector to $\partial \omega$ (which we assume smooth enough for this).

\footnotetext{
${ }^{\dagger}$ The case of pure traction and moments, i.e., $\gamma_{0}$ of null measure, is dealt with by working in the obvious quotient space.
} 
Assume thus that $\varphi \in W^{3, \infty}\left(\omega ; \mathbb{R}^{3}\right)$ and let $v \in W$ be such that $v=0$ on $\gamma_{0}$ and $v$ satisfies condition (60). Then $v_{3}=v \cdot a_{3} \in H^{2}(\omega)$, viz. Lemma 4 , and $\partial_{\alpha} v_{3}=$ $\partial_{\alpha} v \cdot a_{3}+v \cdot \partial_{\alpha} a_{3}$. Hence, $\partial_{\alpha} v_{3}=0$ on $\gamma_{0}$ so that $\partial_{\alpha} v_{3} \nu_{\alpha}=0$ on $\gamma_{0}$, i.e., $v$ satisfies condition (56).

Conversely, assume that $v$ satisfies condition (56) and $v=0$ on $\gamma_{0}$. Then $\partial_{\alpha} v_{3} \nu_{\alpha}=0$ and $\partial_{\alpha} v_{3} \tau_{\alpha}=0$ on $\gamma_{0}$ in the sense of $H^{1 / 2}\left(\gamma_{0}\right)$. Therefore, $\partial_{\alpha} v_{3}=0$ on $\gamma_{0}$ and thus $\partial_{\alpha} v \cdot a_{3}=\partial_{\alpha} v_{3}-v \cdot \partial_{\alpha} a_{3}=0$ on $\gamma_{0}$.

REMARKs. i) Geometrically, the new clamping condition (60) simply means that, at almost every point of $\gamma_{0}$, the tangent plane to the deformed surface remains orthogonal to the original normal vector $a_{3}$.

ii) Convergence results in the spirit of those of Lemma 2 also hold true and are left to the reader. They can serve to further stress that condition (60) is a natural extension of the classical condition.

Let us now consider the infinitesimal rotation vector.

Lemma 13. Assume that $\varphi \in W^{2, \infty}\left(\omega ; \mathbb{R}^{3}\right)$ and let $v \in W$. Then the expression

$$
\psi(v)=\epsilon^{\alpha \beta}\left(\partial_{\beta} v \cdot a_{3}\right) a_{\alpha}+\frac{1}{2} \epsilon^{\alpha \beta}\left(\partial_{\alpha} v \cdot a_{\beta}\right) a_{3}
$$

defines an element of $L^{2}\left(\omega ; \mathbb{R}^{3}\right)$ such that

$$
a^{\alpha} \cdot \psi(v)=\epsilon^{\alpha \beta}\left(\partial_{\beta} v \cdot a_{3}\right) \in H^{1}(\omega) .
$$

Moreover, it coincides with the classical infinitesimal rotation vector (58) if $\varphi \in$ $W^{3, \infty}\left(\omega ; \mathbb{R}^{3}\right)$.

Proof. It is clear that if $v$ belongs to $W$, then expression (62) defines an element of $L^{2}\left(\omega ; \mathbb{R}^{3}\right)$ that satisfies (63). Indeed, $\epsilon^{\alpha \beta} \in W^{1, \infty}(\omega)$. Let us thus assume that $\varphi \in W^{3, \infty}\left(\omega ; \mathbb{R}^{3}\right)$ and let us compare the new expression with the classical expression in this case. We thus have for the tangential components

$$
\begin{aligned}
a^{\alpha} \cdot \psi(v) & =\epsilon^{\alpha \beta}\left(\partial_{\beta} v \cdot a_{3}\right) \\
& =\epsilon^{\alpha \beta}\left(\partial_{\beta} v_{3}-v \cdot \partial_{\beta} a_{3}\right) \\
& =\epsilon^{\alpha \beta}\left(\partial_{\beta} v_{3}+b_{\beta}^{\rho} v_{\rho}\right) .
\end{aligned}
$$

The calculation for the normal component is virtually identical:

$$
\begin{aligned}
2 a^{3} \cdot \psi(v) & =\epsilon^{\alpha \beta}\left(\partial_{\alpha} v \cdot a_{\beta}\right) \\
& =\epsilon^{\alpha \beta}\left(\partial_{\alpha} v_{\beta}-v \cdot \partial_{\alpha} a_{\beta}\right) \\
& =\epsilon^{\alpha \beta}\left(\partial_{\alpha} v_{\beta}-\Gamma_{\alpha \beta}^{\rho} v_{\rho}-b_{\alpha \beta} v_{3}\right) \\
& =\epsilon^{\alpha \beta} v_{\alpha \mid \beta},
\end{aligned}
$$

since $\epsilon^{\alpha \beta} b_{\alpha \beta}=0$.

REMARKS. i) We had already obtained the expression of the normal component of the infinitesimal rotation vector by a direct argument in the inextensional case; see the remarks following Lemma 8.

ii) It is easy to check with expressions (62) and (11) that for all $v \in H^{1}\left(\omega ; \mathbb{R}^{3}\right)$,

$$
\partial_{\alpha} v=\psi(v) \wedge a_{\alpha}+\gamma_{\alpha \beta}(v) a^{\beta}
$$


hence formula (34) holds when $v$ is inextensional.

We now turn to the Koiter problem. Let us define a new space

$$
\widetilde{V}=\left\{v \in H^{1}\left(\omega ; \mathbb{R}^{3}\right), \partial_{\alpha \beta} v \cdot a_{3} \in L^{2}(\omega), v=\partial_{\alpha} v \cdot a_{3}=0 \text { on } \gamma_{0}\right\} .
$$

Note that the condition $\partial_{\alpha \beta} v \cdot a_{3} \in L^{2}(\omega)$ may alternatively be written $\partial_{\alpha} v \cdot a_{3} \in H^{1}(\omega)$. By Lemma 12 , we see that $\widetilde{V}$ is a closed subspace of $W$ that extends the classical space of Bernadou and Ciarlet [2] to the case $\varphi \in W^{2, \infty}\left(\omega ; \mathbb{R}^{3}\right)$. We prove the following existence and uniqueness result.

Theorem 14. Let $P \in L^{2}\left(\omega ; \mathbb{R}^{3}\right), N^{i} \in L^{2}\left(\gamma_{1}\right)$ and $M^{\alpha} \in L^{2}\left(\gamma_{1}\right)$. Then there exists a unique solution of the variational problem: Find $u \in \widetilde{V}$ such that

$$
\forall v \in \widetilde{V}, \quad B(u, v)=l(v)
$$

where

$$
B(u, v)=\int_{\omega} e a^{\alpha \beta \rho \sigma}\left\{\gamma_{\alpha \beta}(u) \gamma_{\rho \sigma}(v)+\frac{e^{2}}{12} \Upsilon_{\alpha \beta}(u) \Upsilon_{\rho \sigma}(v)\right\} \sqrt{a} d x
$$

and

$$
l(v)=\int_{\omega}(P \cdot v) \sqrt{a} d x+\int_{\gamma_{1}}(N \cdot v+M \cdot \psi(v)) \sqrt{a_{\alpha \beta} \tau_{\alpha} \tau_{\beta}} d \sigma
$$

with $N=N^{i} a_{i}$ and $M=M^{\alpha} a_{\alpha} \wedge a_{3}=\epsilon_{\beta \alpha} M^{\alpha} a^{\beta}$.

Proof. Let us first check that the linear form $l$ is continuous on the space $\tilde{V}$. Since the vectors $a_{i}$ belong to $W^{1, \infty}\left(\omega ; \mathbb{R}^{3}\right)$, their trace on $\gamma_{1}$ belongs to $C^{0}\left(\gamma_{1}\right)$. Therefore, if $N^{i} \in L^{2}\left(\gamma_{1}\right)$ and $M^{\alpha} \in L^{2}\left(\gamma_{1}\right)$, then $N \in L^{2}\left(\gamma_{1} ; \mathbb{R}^{3}\right)$ and $M \in L^{2}\left(\gamma_{1} ; \mathbb{R}^{3}\right)$. Moreover, by expression (62), $M \cdot \psi(v)=M^{\rho}\left(\partial_{\rho} v \cdot a_{3}\right)$. Consequently, Lemma 12 and the trace theorem imply that $l$ is continuous on $W$, hence on $\tilde{V}$ (we could have taken $N \in H^{-1 / 2}\left(\gamma_{1} ; \mathbb{R}^{3}\right.$ ) and $\left.M^{\rho} \in H^{-1 / 2}\left(\gamma_{1}\right)\right)$.

Secondly, we show that the bilinear form $B$ is $\widetilde{V}$-elliptic (it is obviously continuous). For this, it suffices to prove that the semi-norm (48) defines a norm on $\tilde{V}$ that is equivalent to the norm of $W$ defined by (26). Assume thus that $v \in \widetilde{V}$ is such that $\|\mid v\| \|=0$. By the infinitesimal rigid displacement lemma, this implies that $v(x)=\psi \wedge \varphi(x)+c$. First of all, $v$ vanishes on $\gamma_{0}$. If $\varphi\left(\gamma_{0}\right)$ is not included in a straight line, it follows that $v=0$ as before. Assume that $\varphi\left(\gamma_{0}\right)$ is included in a straight line $D$ and that $\psi \neq 0$. Then $D$ is parallel to the plane spanned by $\left(a_{\alpha}\right)_{\mid \gamma_{0}}$ and $\psi$ is parallel to $D$. On the other hand, $\partial_{\alpha} v=\psi \wedge a_{\alpha}$ and the clamping condition imply that $\partial_{\alpha} v \cdot a_{3}=a_{\alpha} \wedge a_{3} \cdot \psi=0$. Thus $\psi$ is orthogonal to the plane spanned by $\left(a_{\alpha}\right)_{\mid \gamma_{0}}$, which contradicts the hypothesis. Therefore, $\psi=0$ and $v=0$ on $\gamma_{0}$ then imply that $c=0$.

The rest of the proof concerning the norm equivalence is identical to the proof of Lemma 11.

7. Examples and applications. As we mentioned in the introduction, there exists another formulation of the Koiter model that is valid for $W^{2, \infty}$-midsurfaces. This formulation is due to Destuynder and Salaün $[13,14]$. In this formulation, the infinitesimal rotation vector is not a priori related to the displacement. The relationship between the two is enforced a posteriori by a Lagrange multiplier equal to the transverse shear force. 
Nonetheless, in their derivation, Destuynder and Salaün still assume that the midsurface is of class $C^{3}$. It so happens that the contributions of the derivatives of the second fundamental form cancel out and therefore, the resulting model does not require that these derivatives exist as functions, even though they are used in the derivation of the formulation. Moreover, the existence and uniqueness result for the mixed formulation is based on the fact that the inf-sup condition is satisfied, which is proved by appealing to Bernadou and Ciarlet's ellipticity result, i.e., with a $C^{3}$-midsurface. It would be interesting to see if Destuynder and Salaün's formulation can be rewritten in a more coordinate-free manner so as to allow $W^{2, \infty}$-midsurfaces as well. The fact that the mixed formulation does not require third derivatives of the chart is not too surprising since it uses the displacement and infinitesimal rotation vector as independent variables. It thus resembles Naghdi's model, a model that does not involve third derivatives of the chart; see Blouza [5] for an approach similar to the one used in the present article.

It should be noted that the terms $\partial_{\alpha} b_{\beta}^{\rho} u_{\rho}$ for $u_{\rho} \in H^{1}(\omega)$, which restrict the regularity of the midsurface in the classical approach, actually are distributions in $H^{-1}(\omega)$ in the $W^{2, \infty}$ case. It is thus conceivable that an analysis could be carried out in that case that would yield existence and uniqueness for a formulation in covariant components. However, the variational space cannot be $H^{1} \times H^{1} \times H^{2}$ because the requirement that $\Upsilon_{\alpha \beta}(u)$ be square integrable amounts to asking that $\partial_{\alpha \beta} u_{3}+\partial_{\alpha} b_{\beta}^{\rho} u_{\rho}$ belongs to $L^{2}(\omega)$, which is certainly not the same as $u_{3} \in H^{2}(\omega)$. Our approach incorporates this condition very simply and naturally.

On the numerical side, the mixed formulation is interesting insofar as it only requires $C^{0}$ finite elements and, at the expense of introducing an extra unknown, the infinitesimal rotation vector. In the classical Bernadou and Ciarlet formulation, the natural splitting of the variational space into $H^{1}$ for the tangential components and $H^{2}$ for the normal component implies that conforming methods require $C^{()}$tangential elements together with $C^{1}$ normal elements, such as the Argyris triangle (see Bernadou [1]). In our formulation, this splitting is hidden in the fact that $\partial_{\alpha \beta} u \cdot a_{3}$ belongs to $L^{2}(\omega)$, hence the need for $C^{1}$ (conforming) elements for the whole displacement and not only its normal component, i.e., more degrees of freedom than in the classical formulation. On the other hand, the new formulation requires much less information on the geometry of the midsurface than the classical formulation and assembling the stiffness matrix turns out to be quite a bit simpler. Numerical experiments by Kerdid and Mato-Eiroa [16] show that the new formulation compares well with the classical one on the usual benchmarks. In this respect, it should be pointed out that the new formulation accomodates piecewise $C^{3}$-midsurfaces, which are not usually considered in the literature, entirely naturally; see below for examples. It is also possible to try to lower the computational cost implied by $C^{1}$ elements by using nonconforming DKT type mixed elements; see in this direction Le Tallec and Mani [18].

The simplest and most natural examples of $W^{2, \infty}$-shells are given by globally $C^{1}$ - and piecewise $C^{3}$-midsurfaces. Consider for instance a shell consisting of a planar part that is connected to a circular cylinder part or an egg-shaped shell made of a quarter of a sphere and a quarter of an ellipsoid glued together along a circle (see Fig. 1).

Note that some midsurfaces generated by CAD software packages can also be of this type. In these cases, the derivatives of the second fundamental form contain Dirac masses 


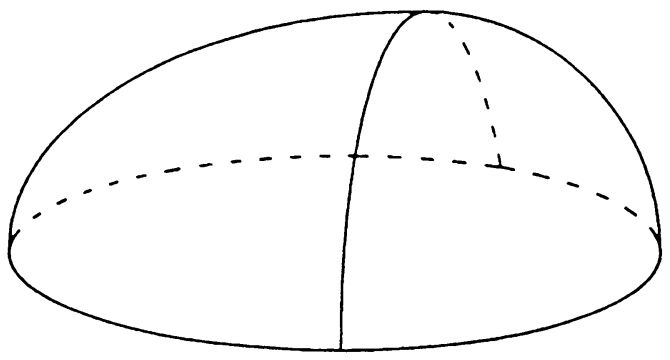

FIG. 1. A simple $W^{2, \infty}$-shell

concentrated on the interfaces between the smooth parts of the shell. The condition $\partial_{\alpha \beta} u \cdot a_{3} \in L^{2}(\omega)$ is easily worked out. Indeed, since it is equivalent to $\partial_{\alpha} u \cdot a_{3} \in H^{1}(\omega)$, this means that the jump of $\partial_{\alpha} u \cdot a_{3}$ vanishes on each interface. In covariant components, this reads $\left[\partial_{\alpha} u_{3}+b_{\alpha}^{\rho} u_{\rho}\right]=0$, or equivalently $\left[\partial_{\alpha} u_{3}\right]=-\left[b_{\alpha}^{\rho}\right] u_{\rho}$, on each interface (with $u_{3}$ piecewise $H^{2}$ ). It is clear that the latter condition cannot easily be numerically implemented, at least in a conforming fashion, whereas the new formulation does not "see" it.

Let us now give another type of application to shells with piezoelectric patches. We consider a shell of constant thickness $e$ to which piezoelectric wafers are bonded. Let us recall the weak formulation of the problem: Find $u \in V$ such that

$$
\forall u \in V, \quad B(u, v)=l(v),
$$

where $B$ is the bilinear form of Koiter's model and the right-hand side is of the form

$$
l(v)=-\frac{h^{\alpha \beta}}{c} \mathcal{V} \int_{\omega_{p}} \gamma_{\alpha \beta}(v) \sqrt{a} d x-(e+t) \frac{h^{\alpha \beta}}{c} \mathcal{V} \int_{\omega_{p}} \Upsilon_{\alpha \beta}(v) \sqrt{a} d x
$$

The open set $\omega_{p}$ is the pre-image in the chart $\varphi$ of the part of the shell where the piezowafer(s) are located. The wafers have piezoelectric tensor $h^{\alpha \beta}$ and capacity $c$ and a voltage $\mathcal{V}$ is applied to them. We assume for simplicity that the chart is such that these coefficients are constant (this is not very important). The wafers are of thickness $2 t$, a parameter that can be much smaller than $e$ in the case of a polymer film for instance. We neglect the stiffness of the wafers compared to that of the shell. Actually, the physically relevant problem is the control problem; in particular, it is a time-dependent problem. To keep a long story short, we only consider here the static problem; see Destuynder and Saïdi [12] for more details.

Under the hypothesis $\varphi \in W^{2, \infty}\left(\omega ; \mathbb{R}^{3}\right)$, the right-hand side (68) remains continuous over $V$ and there is no difficulty in proving existence and uniqueness. The difficulty comes in however when looking for a mechanical interpretation of this right-hand side in terms of applied forces and moments. Indeed, the integrations by parts required for this interpretation can only be performed in the distributional sense in this case, which is not very illuminating. Let us consider the case of a piecewise $C^{3}$-shell. Since the problem is local, it is sufficient to consider a shell made of two $C^{3}$-pieces, separated by an interface 
$\gamma$ across which some curvatures are discontinuous. Moreover, we assume that there is a single wafer bonded on the shell and that it intersects the interface.

Under these hypotheses, the piezoelectric effect amounts to a contribution of several terms. First is a surface force effect on $\omega_{p}$ of the form $\int_{\omega_{p}} g \cdot v \sqrt{a} d x$ with density

$$
g=\frac{h^{\alpha \beta} \mathcal{V}}{c \sqrt{a}}\left(\partial_{\alpha} a_{\beta}(\sqrt{a})-(e+t)\left(\partial_{\alpha \beta}\left(a_{3} \sqrt{a}\right)+\partial_{\rho}\left(\Gamma_{\alpha \beta}^{\rho} a_{3} \sqrt{a}\right)\right)\right)
$$

(here $\partial_{\alpha \beta}\left(a_{3} \sqrt{a}\right)$ and $\partial_{\rho} \Gamma_{\alpha \beta}^{\rho}$ denote the regular part of these quantities. Also note that $\left.\partial_{\alpha}(\sqrt{a})=\left(\Gamma_{\alpha 1}^{1}+\Gamma_{\alpha 2}^{2}\right) \sqrt{a}\right)$. There is also a distribution of forces along the wafer's boundary $\partial \omega_{p}$ of the form $\int_{\partial \omega_{p}} h \cdot v \sqrt{a_{\lambda \mu} \tau_{\lambda} \tau_{\mu}} d \sigma$ with density

$$
h=-\frac{h^{\alpha \beta} \mathcal{V}}{c \sqrt{a_{\lambda \mu} \tau_{\lambda} \tau_{\mu}}}\left(\nu_{\alpha} a_{\beta} \sqrt{a}-(e+t)\left(\nu_{\beta} \partial_{\alpha}\left(a_{3} \sqrt{a}\right)+\nu_{\rho} \Gamma_{\alpha \beta}^{\rho} a_{3} \sqrt{a}\right)\right),
$$

where $\nu_{\alpha}$ are the Cartesian components of the outer normal vector to $\partial \omega_{p}$. Applied moments also arise on $\partial \omega_{p}$ of the form $\int_{\partial \omega_{p}} M \cdot \psi(v) \sqrt{a_{\lambda \mu} \tau_{\lambda} \tau_{\mu}} d \sigma$ with density

$$
M=-\frac{h^{\alpha \beta} \mathcal{V}(e+t)}{c \sqrt{a_{\lambda \mu} \tau_{\lambda} \tau_{\mu}}} \nu_{\beta} \sqrt{a}\left(a_{\alpha} \wedge a_{3}\right)
$$

Finally, the curvature discontinuity manifests itself through a density $h^{\prime}$ of forces concentrated on the interface $\gamma$

$$
h^{\prime}=\frac{h^{\alpha \beta} \mathcal{V}(e+t)}{c \sqrt{a_{\lambda \mu} \tau_{\lambda}^{\prime} \tau_{\mu}^{\prime}}}\left(\nu_{\beta}^{\prime}\left[\partial_{\alpha}\left(a_{3} \sqrt{a}\right)\right]+\nu_{\rho}^{\prime}\left[\Gamma_{\alpha \beta}^{\rho}\right] a_{3} \sqrt{a}\right),
$$

where $\tau^{\prime}$ and $\nu^{\prime}$ are tangent and normal unit vectors on the interface.

Acknowledgments. The authors would like to thank the anonymous referee for making constructive remarks that helped greatly improve the manuscript.

\section{REFERENCES}

[1] M. Bernadou, Méthodes d'éléments finis pour les problèmes de coques minces, RMA, Masson, Paris, 1994

[2] M. Bernadou and P. G. Ciarlet, Sur l'ellipticité du modèle linéaire de coques de W. T. Koiter, in Computing Methods in Applied Sciences and Engineering (Second Internat. Sympos., Versailles, 1975), R. Glowinski and J.-L. Lions (eds.), Lecture Notes in Economics and Math. Systems, vol. 134, Springer-Verlag, Berlin, 1976, pp. 89-136

[3] M. Bernadou, P. G. Ciarlet, and B. Miara, Existence theorems for two-dimensional linear shell theories, J. Elasticity 34, 111-138 (1994)

[4] A. Blouza, Existence et unicité pour le modèle de Naghdi pour une coque peu régulière, C. R. Acad. Sci. Paris 324, Série I, 839-844 (1997)

[5] A. Blouza and H. Le Dret, Sur le lemme du mouvement rigide, C. R. Acad. Sci. Paris 319, Série I, 1015-1020 (1994)

[6] A. Blouza and H. Le Dret, Existence et unicité pour le modèle de Koiter pour une coque peu régulière, C. R. Acad. Sci. Paris 319, Série I, 1127-1132 (1994)

[7] D. Choi, Rigidité infinitésimale d'un type de pli d'une surface en déplacement inextensionnel, C. R. Acad. Sci. Paris 317, Série I, 323-327 (1993)

[8] D. Choi and E. Sanchez-Palencia, Rigidification de surfaces réglées par des plis. Application à la statique et à la dynamique des coques élastiques minces, C. R. Acad. Sci. Paris 317, Série I, 415-422 (1993) 
[9] P. G. Ciarlet and B. Miara, On the ellipticity of linear shell models, Z. Angew. Math. Phys. 43, 243-253 (1992)

[10] E. Cosserat and F. Cosserat, Théorie des corps déformables, Hermann, Paris, 1909

[11] P. Destuynder, Sur une justification des modèles de plaques et de coques par les méthodes asymptotiques, Doctoral dissertation, Université Pierre et Marie Curie, Paris, 1980

[12] P. Destuynder and A. Saïdi, Smart materials and flexible structures, to appear in European Journal of Mechanics, A/Solids

[13] P. Destuynder and M. Salaün, Une formulation variationnelle mixte pour les modèles généraux de coques minces, C. R. Acad. Sci. Paris 310, Série I, 215-220 (1990)

[14] P. Destuynder and M. Salaün, A mixed formulation for a general shell model, Mat. Apl. Comput. 10, 161-190 (1991)

[15] G. Geymonat and E. Sanchez-Palencia, On the rigidity on certain surfaces with folds and applications to shell theory, Arch. Rational Mech. Anal. 129, 11-45 (1995)

[16] N. Kerdid and P. Mato-Eiroa, Conforming finite element approximation for shells with little regularity, to appear

[17] W. T. Koiter, On the foundations of the linear theory of thin elastic shells, Proc. Kon. Ned. Akad. Wetensch. B73, 169-195 (1970)

[18] P. Le Tallec and S. Mani, Analyse numérique d'un modèle de coques de Koiter discrétisé en base cartésienne par éléments finis DKT, RAIRO Modél. Math. Anal. Numér. 32, $433-450$ (1998)

[19] P. Rougée, Équilibre des coques élastiques minces inhomogènes en théorie non linéaire, Doctoral dissertation, Université de Paris, 1969

[20] R. Valid, La mécanique des milieux continus et le calcul des structures, Eyrolles, Paris, 1977

[21] I. N. Vekua, Generalized Analytic Functions, Pergamon Press, Oxford, 1962 